\title{
(6) OPEN ACCESS \\ Enter B and W: two new meningococcal vaccine programmes launched
}

\author{
Shamez N Ladhani, ${ }^{1}$ Mary Ramsay, ${ }_{1}^{1}$ Ray Borrow, ${ }^{2}$ Andrew Riordan, ${ }^{3}$ \\ John M Watson, ${ }^{4}$ Andrew J Pollard ${ }^{5}$
}

${ }^{1}$ Immunisation Department, Public Health England, London, UK

${ }^{2}$ Vaccine Evaluation Unit, Public Health England, Manchester Royal Infirmary, Manchester, UK

${ }^{3}$ Department of Infectious Diseases and Immunology, Alder Hey Children's Hospital, Liverpool, UK

${ }^{4}$ Department of Health, London, UK

${ }^{5}$ Department of Paediatrics, University of Oxford, Children's Hospital, Oxford, UK

\section{Correspondence to} Professor Andrew J Pollard, Department of Paediatrics, University of Oxford, Level 2, Children's Hospital, Oxford OX3 9DU, UK; andrew. pollard@paediatrics.ox.ac.uk

Received 25 August 2015 Accepted 23 October 2015
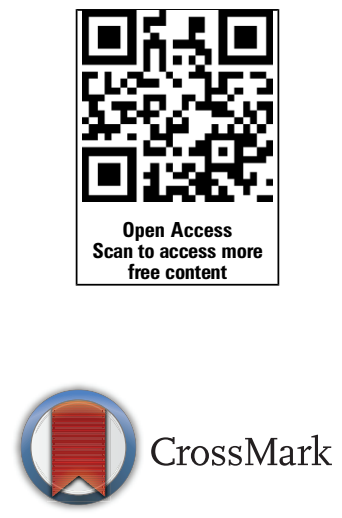

To cite: Ladhani $S N_{\text {, }}$ Ramsay M, Borrow R, et al. Arch Dis Child

2016;101:91-95.

\section{ABSTRACT}

In 2015, the UK became the first country in the world to have a comprehensive routine meningococcal vaccine programme targeting all of the main capsular groups of $N$. meningitidis. ${ }^{1}$ An infant vaccine programme against meningococcal capsular group B Neisseria meningitidis (MenB) was launched from 1st September with an aim to reduce endemic MenB disease in early childhood. On 1st August 2015, an adolescent programme against groups $A, C, W$ and $Y$ meningococci (MenACWY) was rolled out to halt a growing outbreak of capsular group W disease (MenW) caused by a hypervirulent clone of N. meningitidis, in addition to maintaining control against MenC disease provided by the current adolescent programme. $^{2}$

\section{INTRODUCTION}

Meningococcal disease is the leading infectious cause of death in early childhood, ${ }^{3}$ and its control has been a public health priority for decades. In the UK, most cases have been caused by MenB and MenC, with small numbers caused by MenW and MenY; other capsular groups are rare (figure 1). During the 1990 s, a rapid rise in cases caused by a single MenC clone led to the introduction of the National Health Service (NHS) MenC vaccination programme in 1999 , which successfully controlled the outbreak and has sustained population protection against MenC over the past 15 years; it is now apparent that the success of this programme depends largely on indirect (herd) protection. ${ }^{4}$ Meningococci are carried in the nasopharynx of healthy individuals, particularly adolescents and young adults, and only cause disease when transmitted to susceptible individuals. MenC vaccines are made by chemical conjugation of the surface capsular polysaccharide to a carrier protein-such as tetanus toxoid or a mutant diphtheria toxoid (CRM197)—making a highly immunogenic construct. MenC conjugate vaccines provide individual protection against MenC disease and prevent acquisition of colonisation in the nasopharynx, thereby interrupting transmission of $\mathrm{MenC}$ so that the wider population is protected. Adjustments to the MenC programme in recent years have focused on enhancing immunity among teenagers to prevent colonisation and thus enhance indirect population protection as carriage of meningococci is highest among adolescents.

Since the success of the MenC conjugate vaccination programme, MenB has been responsible for almost all cases of invasive meningococcal disease in the UK, especially in children and young adults. In
England, as in the rest of Europe, MenB cases have been declining across all age groups: from 1614 cases in the 2000/2001 epidemiological year (July 2000 to June 2001) to 418 cases in 2014/2015 (figure 1). This decline is most likely due to natural secular trends and MenB cases are expected to increase again at some point in the future (as seen with MenB and MenC in the mid-1990s), due to, for example, the introduction of a new MenB strain into the population. The burden of meningococcal disease is greatest in young children with half the cases currently diagnosed in those less than 5 years of age. Infants ( $<1$ year-old) have the highest number of cases and deaths due to MenB. ${ }^{5}$ After the 1 st year of life, cases decline with age until adolescence where a smaller peak is observed (figure 2).

Development of vaccines against MenB has been difficult since its polysaccharide capsule is identical to saccharides which decorate fetal neural cell adhesion molecules and are, therefore, poorly immunogenic self-antigens. ${ }^{6}$ Previous attempts at making meningococcal vaccines using subcapsular antigens have been hampered by poor immunogenicity and/or antigenic variability of the antigens, which has limited progress in the development of vaccines against highly diverse endemic MenB strains. Fifteen years ago, however, the first meningococcal genome sequence was used to predict meningococcal surface antigens and to construct three components of the four-component MenB vaccine, $4 \mathrm{CMenB}$. The fourth component of the vaccine is an outer membrane vesicle (OMV) made by detergent extraction of meningococcal OMVs from a strain causing an extended outbreak in New Zealand. This latter component has been used as a stand-alone vaccine and successfully controlled the epidemic in New Zealand. ${ }^{7}$ When combined with the other three components, $4 \mathrm{CMenB}$ is predicted to cover $73-88 \%$ of MenB strains causing invasive disease in the UK. 89

After more than a decade of clinical development, $4 \mathrm{CMenB}$ was licensed by the European Medicines Agency in early 2013. The potential use of the vaccine in the UK was considered by the Department of Health's Joint Committee on Vaccination and Immunisation (JCVI). ${ }^{10}$ The deliberations considered the safety and efficacy of the vaccine and noted the high case fatality ratio (CFR) and sequelae rate of the disease, highlighting the importance of controlling meningococcal disease for child health in the UK. The cost-effectiveness analysis for this relatively uncommon disease showed that the vaccine would only be costeffective at a low vaccine price, ${ }^{11}$ and JCVI 
Figure 1 Laboratory-confirmed cases of invasive meningococcal disease in England by capsular group and epidemiological year. The blue arrow denotes the start of the national immunisation programme against group $\mathrm{C}$ meningococcal (MenC) disease. Source: Public Health England.

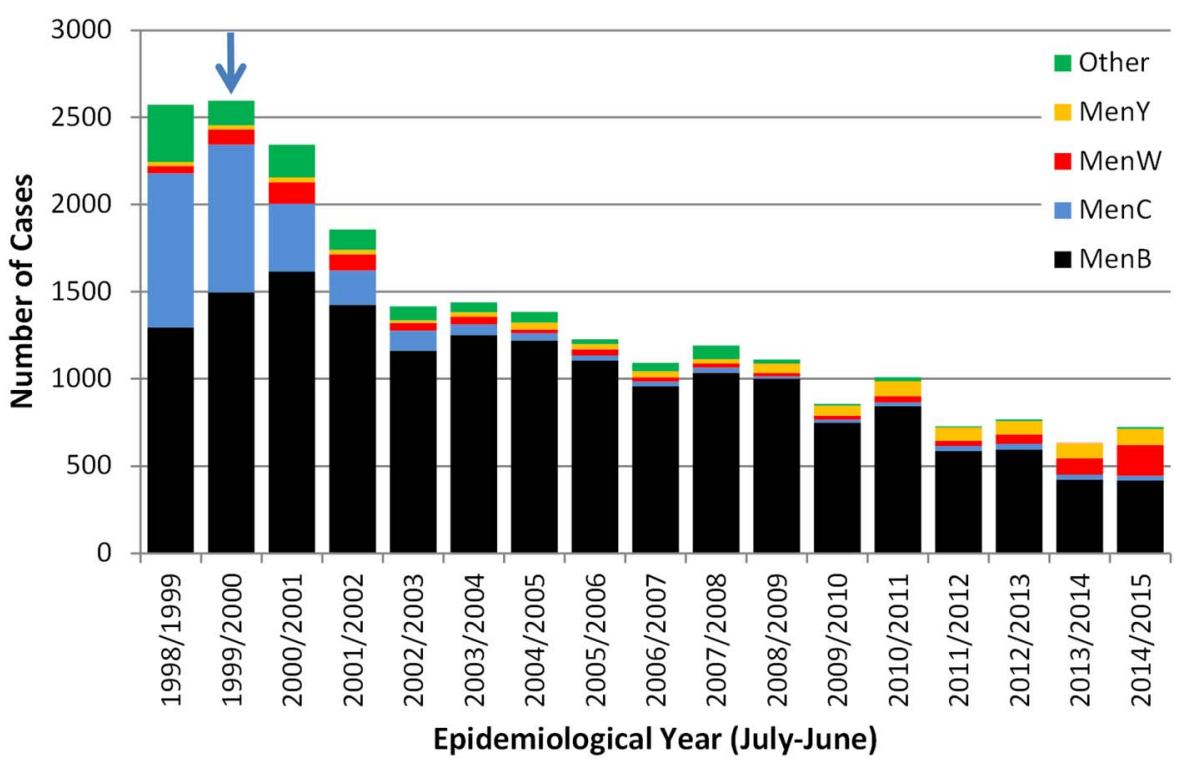

recommended the vaccine for introduction if such a price could be met. The negotiations with the manufacturer took a year to conclude and a programme was announced in June $2015 .{ }^{1}$

This much anticipated and ground-breaking programme commenced in autumn 2015, with a three-dose schedule of $4 \mathrm{CMenB}$ to be offered as part of the primary immunisation schedule at 2 months, 4 months and 12 months of age. When $4 \mathrm{CMenB}$ is administered with other routine infant immunisations, fever occurs in $51-61 \%$ of infants. ${ }^{12}$ Despite previous advice that paracetamol might interfere with the immune response to infant vaccines, a recent clinical trial showed that prophylactic paracetamol significantly reduces the rate and intensity of fever after vaccination with 4CMenB, without affecting the immune responses to any of the infant vaccines. ${ }^{13}$ Consequently, parents will be advised to give three doses of paracetamol when their infants receive $4 \mathrm{CMenB}$ as part of the infant schedule, with the first dose given with or as soon as possible after vaccination. Paediatricians should be aware that, in clinical trials, some postvaccination fevers resulted in attendance at medical facilities. A careful history of recent vaccination should be elicited; fever due to vaccination peaks at around $6 \mathrm{~h}$ and is unlikely to persist beyond $48 \mathrm{~h}$. As with all fevers in infants, the potential for serious bacterial infection should be considered, even when temporally related to the vaccine.
Like MenC, 4CMenB was licensed on the basis of immunogenicity, although the OMV component has been shown to be efficacious against matching strains in New Zealand. A specific laboratory assay known as Meningococcal Antigen Typing System was developed as proxy for the existing correlate of protection, and this was used to predict the proportion of invasive MenB disease that might be preventable by $4 \mathrm{CMenB} .{ }^{8}$ Consequently, there remains uncertainty about the precise level, breadth and duration of protection that will be induced by the new programme. The MenB implementation will be closely monitored by Public Health England with detailed follow-up of all laboratory-confirmed cases in children younger than 5 years. Acute and convalescent sera will be requested to assess immune responses to meningococcal disease in vaccinated and unvaccinated children. At the same time, meningococcal isolates and PCR-positive clinical samples will undergo genetic and molecular testing to determine whether or not they were potentially preventable by $4 \mathrm{CMenB}$. The UK is, therefore, likely to be the first country to be able to determine vaccine effectiveness in the field. A detailed Public Health England surveillance plan for England is available online. ${ }^{1}$ The programme will be reviewed by JCVI and it is expected that the first reliable indications will become available around 2 years after implementation. The infant programme is expected to provide direct protection for
Figure 2 Age distribution of laboratory-confirmed invasive meningococcal cases by capsular group in children and young adults over the past 10 years (England, 2004/ 2005 to $2014 / 2015$ ).

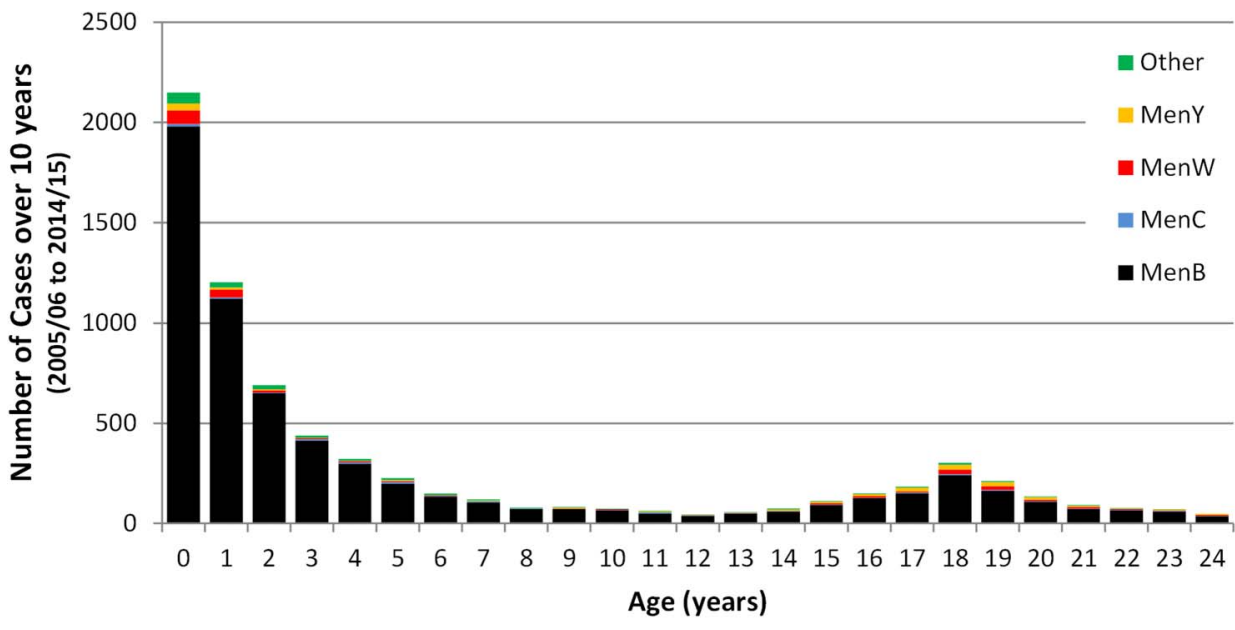


vaccinated children but will provide limited indirect (herd) protection as infants are not thought primarily responsible for carriage and transmission of meningococci. Further work is being undertaken to assess the potential for an adolescent MenB programme that, like the MenC immunisation programme, might induce direct and indirect protection, but requires large-scale carriage studies and several years to gather the information required to support such a programme.

Rates of meningococcal disease vary over time as a result of complex interactions between population immunity and the meningococcal bacteria, driven by the arrival and eventual disappearance of various clones with differing potential for causing disease. During the 1990s, a specific clone, part of the ST-11 complex, and bearing the MenC polysaccharide capsule, caused a large increase in invasive disease and was associated with a high CFR. The response to this increase was the accelerated development and introduction of the MenC vaccine. ${ }^{14}$ The subsequent control of MenC disease in the UK has acted as a model for other countries and for other vaccines, including the recent deployment of conjugate vaccination against group $\mathrm{A}$ meningococci in Africa. ${ }^{15}$

Until recently in the UK, MenW has been a rare cause of meningococcal disease, accounting for less than $5 \%$ of all cases across all age groups. ${ }^{5}$ In the early 2000s, an international outbreak of MenW disease associated with pilgrimage to the Hajj was terminated by the introduction of mandatory MenACWY vaccination for all pilgrims. ${ }^{16}$ Since 2009 , however, there has been a year-on-year increase in the number of MenW cases in England, from 19 in the 2008/2009 epidemiological year to 176 in 2014/2015, accounting for a quarter of all laboratoryconfirmed meningococcal cases in that year (figure 3). The emergent MenW strain belongs to the highly virulent ST-11 clonal complex that was responsible for the UK MenC outbreak in the 1990s and is genetically identical to MenW strains currently causing large national outbreaks in South America. ${ }^{2}$ In England, MenW cases are being diagnosed across all regions and all age groups. Most MenW cases are diagnosed in previously healthy individuals who have not travelled abroad, indicating that this strain is now established as endemic, and who may develop severe clinical disease often requiring intensive care support. Moreover, the clinical presentation has sometimes been atypical, with septic arthritis and severe respiratory tract infections (including pneumonia, epiglottitis and supraglottitis) being over-represented among MenW cases compared with other meningococcal groups. Several adolescents and young adults

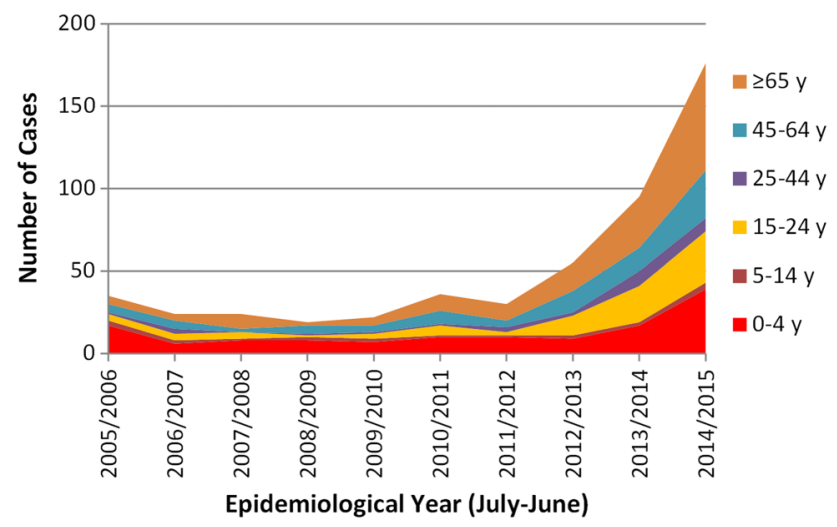

Figure 3 Laboratory-confirmed cases of invasive meningococcal group W (MenW) disease by age group and epidemiological year in England. with MenW septicaemia presented primarily with gastrointestinal symptoms and progressed rapidly to death without having the characteristic non-blanching rash. A consequence of such atypical presentations is that meningococcal disease is not considered in the differential diagnosis until a positive culture becomes available, potentially leading to delays in diagnosis, offering chemoprophylaxis to close contacts and identifying clusters and local outbreaks.

The alarming rise in MenW cases probably reflects the virulence properties of the ST-11 clonal complex. In South America, the MenW CFR was 27\%. ${ }^{17}$ In the UK, since its arrival, MenW CFR has been more than double (12\%) that seen with MenB disease over the same period $(<5 \%)$, and similar to that observed with the MenC ST-11 clonal complex outbreak in the UK in the 1990 s. $^{18}$

Fortunately an effective vaccine for MenW already exists as part of multicomponent conjugate vaccines that also protect against group A, C, W and Y meningococci (MenACWY). Like the MenC conjugates, these vaccines contain the capsular polysaccharides of these four meningococcal capsular groups chemically conjugated to tetanus toxoid, CRM197 or diphtheria toxoid (diphtheria toxoid conjugate only available in the USA). In February 2015, the Department of Health accepted JCVI's advice that the vaccine should be used to control the rapid increase in the number of MenW cases. From 1st August 2015, the MenACWY conjugate vaccine will be offered to adolescents aged 14-18 years because this age group experiences a high attack rate, and, as with MenC, teenagers and young adults have the highest carriage rates and are considered to be responsible for driving transmission. ${ }^{19}$ The JCVI considered it highly likely that vaccinating individuals in this age group will result in wide population protection and eventual control of MenW. Because the MenACWY conjugate vaccine also protects against MenC disease, it will replace the MenC conjugate vaccine currently in the routine adolescent programme from late 2015 to maintain long-term population control of MenC.

The adolescent MenACWY conjugate vaccine will be introduced as rapidly as possible given the constraints in vaccine supply. The whole 14-18 year old cohort should be offered the vaccine during 2015-2017 (table 1). The proposed programme will:

- Prioritise the current school year 13-year-olds (17-18 year olds) for vaccination through general practice in order to protect those entering higher education settings such as universities in the next academic year because 1st year undergraduate students, especially those living in university halls of residence, are at significantly higher risk of meningococcal disease than non-students. ${ }^{20} 21$

- Replace MenC with MenACWY vaccination in the existing time-limited 'freshers' programme (ie, for older first-time university entrants who have not already received MenACWY through school year 13) through general practice.

- Replace MenC with MenACWY vaccination in the routine adolescent schools programme (school year 9 or 10) from autumn 2015.

- Include a catch-up campaign for current school year 10 students through schools from January 2016.

- Plan subsequent catch-up campaigns to cover the current school years 11 and 12 when these students reach year 13 .

The introduction of the MenB vaccine for infants may also provide some protection against MenW, since one of the components of $4 \mathrm{CMenB}$ vaccine (neisserial adhesion $\mathrm{A}, \mathrm{NadA}$ ) is found on the surface of the emergent MenW ST-11 clonal 
Table 1 Summary of the adolescent groups A, C, W and Y meningococci (MenACWY) conjugate vaccination programme in (A) areas that currently offer the group $C$ meningococcal (MenC) conjugate vaccine to school year 9 and (B) that currently offer the MenC conjugate vaccine to school year 10 while working towards a transition to a year 9 meningococcal vaccination programme in 2016/2017.

\begin{tabular}{|c|c|c|c|c|c|c|}
\hline \multirow[b]{2}{*}{ Birth cohort } & \multirow{2}{*}{$\begin{array}{l}2014 / 2015 \\
\text { year-age }\end{array}$} & \multicolumn{5}{|c|}{ Academic year } \\
\hline & & $2014 / 2015$ & $2015 / 2016$ & $2016 / 2017$ & $2017 / 2018$ & $2018 / 2019$ \\
\hline \multicolumn{7}{|c|}{ (a) For areas routinely vaccinating in school year 9} \\
\hline 01/09/2003-31/08/2004 & Y6-10/11 & & & & Y9 ACWY & \\
\hline 01/09/2002-31/08/2003 & Y7-11/12 & & & Y9 ACWY & & \\
\hline 01/09/2001-31/08/2002 & Y8-12/13 & & Y9 ACWY & & & \\
\hline 01/09/2000-31/08/2001 & Y9-13/14 & Y9 MenC & & Y11 ACWY & & \\
\hline 01/09/1999-31/08/2000 & $\mathrm{Y} 10-14 / 15$ & & Y11 ACWY & & & \\
\hline \multicolumn{7}{|c|}{ (b) For areas routinely vaccinating in school year 10, working towards a transition to year 9 in 2016/17 } \\
\hline $01 / 09 / 2003-31 / 08 / 2004$ & $Y 6-10 / 11$ & & & & Y9 ACWY & \\
\hline $01 / 09 / 2002-31 / 08 / 2003$ & $Y 7-11 / 12$ & & & Y9 ACWY & & \\
\hline 01/09/2001-31/08/2002 & $Y 8-12 / 13$ & & & Y10 ACWY & & \\
\hline 01/09/2000-31/08/2001 & Y9-13/14 & & Y10 ACWY & & & \\
\hline 01/09/1999-31/08/2000 & $\mathrm{Y} 10-14 / 15$ & Y10 MenC & Y11 ACWY & & & \\
\hline
\end{tabular}

complex (unpublished observations). The MenB programme may, therefore, provide some protection for infants and toddlers until population control is established through the herd protection engendered by the adolescent programme.

Currently, the MenC conjugate vaccination programme consists of a dose at 3 months, 12 months (in a Haemophilus influenzae type b/MenC combination) and 13-14 years of age. Once the MenACWY programme and MenB programmes are fully established, it may be possible to drop the infant dose at 3 months because MenC disease is rare in infants, the adolescent MenACWY programme will maintain herd protection for the population and the infant MenB programme may also offer some protection against other meningococcal capsular groups, including MenC. The current programme with these recent changes can be found here: (https://www.gov.uk/government/uploads/ system/uploads/attachment_data/file/465880/9406_PHE_ 2015_Routine_Childhood_Immunisation_Schedule_12.p $\overline{d f}$ ).

Because of the established enhanced national surveillance in place to monitor the MenC programme over the past two decades, the $\mathrm{UK}$ is in a unique position to evaluate the impact of both new programmes. The implementation of a comprehensive programme against capsular groups A, B, C, W and Y meningococci is a huge step forward for child health in the UK. While the full extent will not be known for several years, these programmes have the potential to have a major impact on the incidence of meningococcal disease across the whole population.

\section{More information}

Meningococcal B (MenB) vaccination programme

Public Health England. https://www.gov.uk/government/ collections/meningococcal-b-menb-vaccination-programme (First published 30 June 2015)

Joint Committee on Vaccination and Immunisation, 2014 https://www.gov.uk/government/uploads/system/uploads/ attachment_data/file/294245/JCVI_Statement_on_MenB.pdf

Contributors All of the authors contributed equally to the development and final review of the manuscript.
Competing interests AJP is Chair of the Department of Health's Joint Committee on Vaccination and Immunisation. AJP has previously conducted research on meningococcal vaccines on behalf of the University of Oxford funded by vaccine manufacturers but no longer does so, and has not received any personal payments from manufacturers. RB performs contract research on behalf of Public Health England for GSK, Novartis, Pfizer and Sanofi Pasteur but receives no personal remuneration. SNL performs contract research on behalf of Public Health England and St. George's University of London for vaccine manufacturers but receives no personal remuneration. JMW is the deputy chief medical officer at the Department of Health.

Provenance and peer review Commissioned; externally peer reviewed.

Open Access This is an Open Access article distributed in accordance with the Creative Commons Attribution Non Commercial (CC BY-NC 4.0) license, which permits others to distribute, remix, adapt, build upon this work non-commercially, and license their derivative works on different terms, provided the original work is properly cited and the use is non-commercial. See: http://creativecommons.org/ licenses/by-nc/4.0/

\section{REFERENCES}

1 Public Health England. Meningococcal B (MenB) vaccination programme. 2015. https://www.gov.uk/government/publications/menb-vaccination-introduction-from-1september-2015

2 Lucidarme J, Hill DMC, Bratcher HB, et al. Genomic resolution of an aggressive, widespread, diverse and expanding meningococcal serogroup $B, C$ and $W$ lineage. J Infect 2015;71:544-52.

3 Ladhani S, Pebody RG, Ramsay ME, et al. Continuing impact of infectious diseases on childhood deaths in England and Wales, 2003-2005. Pediatr Infect Dis J 2010;29:310-13.

4 Campbell $\mathrm{H}$, Andrews N, Borrow R, et al. Updated postlicensure surveillance of the meningococcal $C$ conjugate vaccine in England and Wales: effectiveness, validation of serological correlates of protection, and modeling predictions of the duration of herd immunity. Clin Vaccine Immunol 2010;17:840-7.

5 Ladhani SN, Flood JS, Ramsay ME, et al. Invasive meningococcal disease in England and Wales: implications for the introduction of new vaccines. Vaccine 2012;30:3710-16.

6 Tan LK, Carlone GM, Borrow R. Advances in the development of vaccines against Neisseria meningitidis. N Engl J Med 2010;362:1511-20.

7 Arnold R, Galloway Y, McNicholas A, et al. Effectiveness of a vaccination programme for an epidemic of meningococcal $B$ in New Zealand. Vaccine 2011;29:7100-6.

8 Vogel U, Taha MK, Vazquez JA, et al. Predicted strain coverage of a meningococcal multicomponent vaccine (4CMenB) in Europe: a qualitative and quantitative assessment. Lancet Infect Dis 2013;13:416-25.

9 Frosi G, Biolchi A, Lo Sapio M, et al. Bactericidal antibody against a representative epidemiological meningococcal serogroup $B$ panel confirms that MATS underestimates 4CMenB vaccine strain coverage. Vaccine 2013;31: 4968-74.

10 Joint Committee on Vaccination and Immunisation (JCVI). JCVI position statement on use of Bexsero $®$ meningococcal $B$ vaccine in the UK. 2014. https://www.gov. uk/government/publications/meningococcal-b-vaccine-jcvi-position-statement 
11 Christensen $\mathrm{H}$, Trotter $\mathrm{CL}$, Hickman M, et al. Re-evaluating cost effectiveness of universal meningitis vaccination (Bexsero) in England: modelling study. BMJ 2014;349:95725.

12 Gossger N, Snape MD, Yu LM, et al. Immunogenicity and tolerability of recombinant serogroup $B$ meningococcal vaccine administered with or without routine infant vaccinations according to different immunization schedules: a randomized controlled trial. JAMA 2012;307:573-82.

13 Prymula R, Esposito S, Zuccotti GV, et al. A phase 2 randomized controlled trial of a multicomponent meningococcal serogroup B vaccine (I). Hum Vaccin Immunother 2014;10:1993-2004.

14 Miller E, Salisbury D, Ramsay M. Planning, registration, and implementation of an immunisation campaign against meningococcal serogroup $C$ disease in the UK: a success story. Vaccine 2001;20(Suppl 1):S58-67.

15 Daugla DM, Gami JP, Gamougam K, et al. Effect of a serogroup A meningococcal conjugate vaccine (PSA-TT) on serogroup A meningococcal meningitis and carriage in Chad: a community study [corrected]. Lancet 2014;383:40-7.
16 Hahné SJ, Gray SJ, Jean-François, et al. W135 meningococcal disease in England and Wales associated with Hajj 2000 and 2001. Lancet 2002; 359:582-3.

17 Valenzuela MT, Moreno G, Vaquero A, et al. [Emergence of W135 meningococcal serogroup in Chile during 2012]. Rev Med Chil 2013;141: $959-67$.

18 Ladhani SN, Beebeejaun K, Lucidarme J, et al. Increase in endemic Neisseria meningitidis capsular group W ST-11 complex associated with severe invasive disease in England and Wales. Clin Infect Dis 2015;60:578-85.

19 Christensen H, May M, Bowen L, et al. Meningococcal carriage by age: a systematic review and meta-analysis. Lancet Infect Dis 2010;10: 853-61.

20 Bruce MG, Rosenstein NE, Capparella JM, et al. Risk factors for meningococcal disease in college students. JAMA 2001;286:688-93.

21 Nelson SJ, Charlett A, Orr HJ, et al. Risk factors for meningococcal disease in university halls of residence. Epidemiol Infect 2001;126:211-17. 